\title{
A Wirelessly-Powered Electro-Acupuncture based on Adaptive Pulse Width Mono-Phase Stimulation
}

\author{
Kiseok Song, Seulki Lee, and Hoi-Jun Yoo \\ Department of Electrical Engineering \\ KAIST \\ Daejeon, Republic of Korea \\ sks8795@eeinfo.kaist.ac.kr
}

\begin{abstract}
A wirelessly-powered electro-acupuncture (EA) that dynamically adapts to body-impedance variation (BIV), is proposed. The proposed EA consists of a slender needle, a helical antenna (70 turns, $1 \mathrm{~mm}$ diameter) using conductive yarn $(100 \mu \mathrm{m}$ diameter, $6.6 \Omega / \mathrm{m})$, and a $1.56 \mathrm{~mm}^{2}$ stimulator chip fabricated in $0.18 \mu \mathrm{m} \mathrm{1P6M} \mathrm{CMOS} \mathrm{process.} \mathrm{A} \mathrm{stable} \mathrm{supply}$ voltage of $1 \mathrm{~V}$ is wirelessly generated from $433 \mathrm{MHz}-\mathrm{ISM}$ band with sensitivity of $-16 \mathrm{dBm}$. To deal with BIV in the range of $100 \mathrm{~K} \Omega-200 \mathrm{k} \Omega$, Adaptive-Pulse-Width (APW) scheme is introduced to maintain constant charge injection of $80 \mathrm{nC}$ per stimulation. A pair of EAs forms an EA node, and they operate in Alternate Mono-Phase (AMP) fashion to guarantee the safety by neutralization of the injected charge.
\end{abstract}

\section{INTRODUCTION}

$\mathrm{R}$ ecently, medical treatments using acupuncture/needle especially with electrical current, namely the Electroacupuncture (EA), have been widely used for myalgia [1], infertility [2], and anesthesia treatments [3]. In the conventional EA system shown in Fig. 1 (a), patients are stimulated by needles which are all cumbersomely wireconnected to a bulky EA base station. Although multi-channel EA treatment [4] (up to 6-channel) enhances the potential remedial value of EA, increasing the number of channel results in degradation of user-convenience. It causes slender wired-needles to be easily bent or pulled out even by patient's slight movement. Consequently, the patient feels a huge stress as well as the remedial value degradation. To achieve both high remedial value and patient's convenience simultaneously, this paper presents a wirelessly-powered EA based on Adaptive Pulse Width (APW) Mono-Phase stimulation as shown in Fig. 1 (b). The wirelessly-powered EA with zerothreshold NMOS rectifier eliminates the cumbersome wire connection between EAs and the EA base station so that it both reduces the complexity of the system significantly and increases the number of stimulation channels.

Design of wirelessly-powered EA, has two essential challenges: 1) constant amounts of charge must be injected insitu for effective treatment [4], and 2) the injected charge into the body must be neutralized for patient's safety. According to our measurement results, either penetration depth of the needle into the body or acupuncturing site of the body causes bodyimpedance variation (BIV) from $100 \mathrm{k} \Omega$ to $200 \mathrm{k} \Omega$. A voltage stimulator [5] cannot inject constant charge; since the stimulation current level varies subject to such wide range of BIV. On the contrary, a current stimulator [6] can inject constant charge regardless of BIV. But, it consumes too much power to be adopted in wirelessly-powered EA system. To accommodate to BIV during treatments, an APW stimulation scheme is proposed for maintaining the constant charge injection. Meanwhile, in order to guarantee the safety of using the proposed EA under wirelessly-powered environments, an Alternate Mono-Phase (AMP) scheme is introduced to neutralize the amounts of injected charge by a pair of EAs.

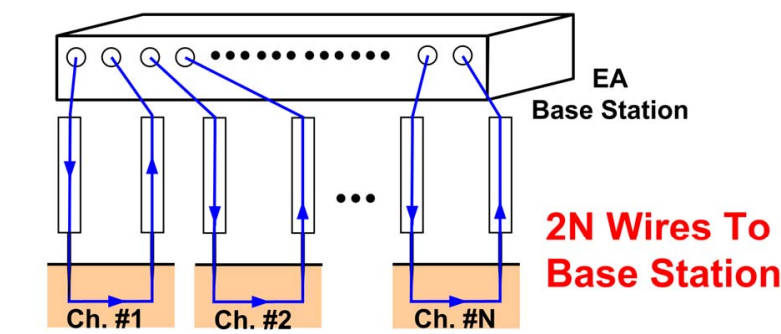

(a)

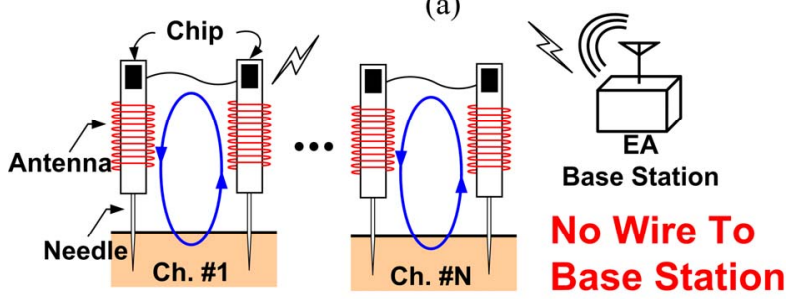

(b)

Figure 1. (a) Conventional and (b) proposed EA system

The rest of the paper is organized as follows. In Section II, the structure and operation of the proposed EA will be discussed. Section III describes the APW and AMP scheme, and Section IV shows the implementation results are given. Finally, Section V concludes the paper. 


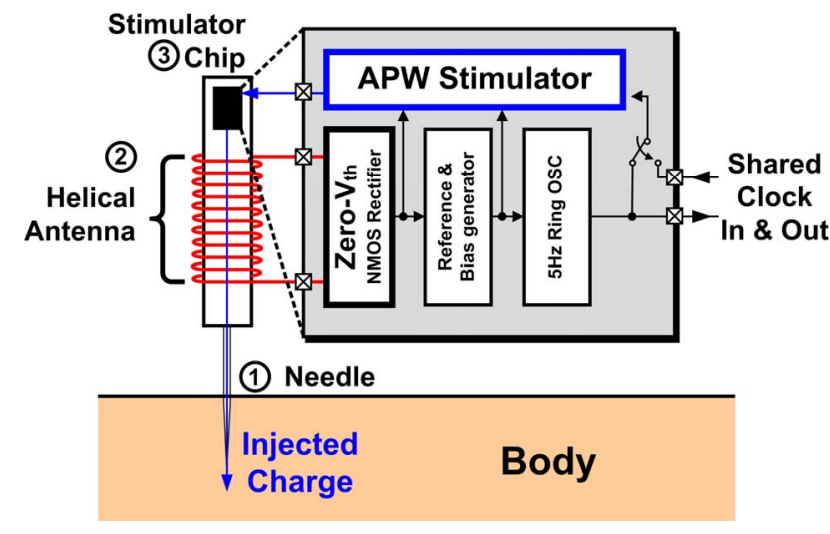

Figure 2. The structure of the proposed wirelessly-powered EA

\section{THE STRUCTURE AND OPERATION OF PROPOSED WIRELEESLY-POWERED EA}

Fig. 2 shows the structure of the proposed wirelesslypowered EA. It consists of a stainless steel needle $(0.25 \mathrm{~mm}$ diameter, $50 \mathrm{~mm}$ length), a helical antenna (70 turns, $1 \mathrm{~mm}$ diameter) and the proposed stimulator chip. The wirelesslypowered EA is implemented by the stimulator chip integrated on the top side of needle, a conductive yarn $(100 \mu \mathrm{m}$ diameter, $6.6 \Omega / \mathrm{m})[8]$ coiled around needle to form a power receiving helical antenna, and a needle tip of the bottom side to inject constant charge provided from APW stimulator. Although the power receiving antenna suffers from variation of resonant frequency up to $9.63 \%$, its matching with stimulator chip is acceptable, because the $\mathrm{S}_{11}$-parameter at $433 \mathrm{MHz}-\mathrm{ISM}$ band is below $-10 \mathrm{~dB}$ as shown in Fig. 3.

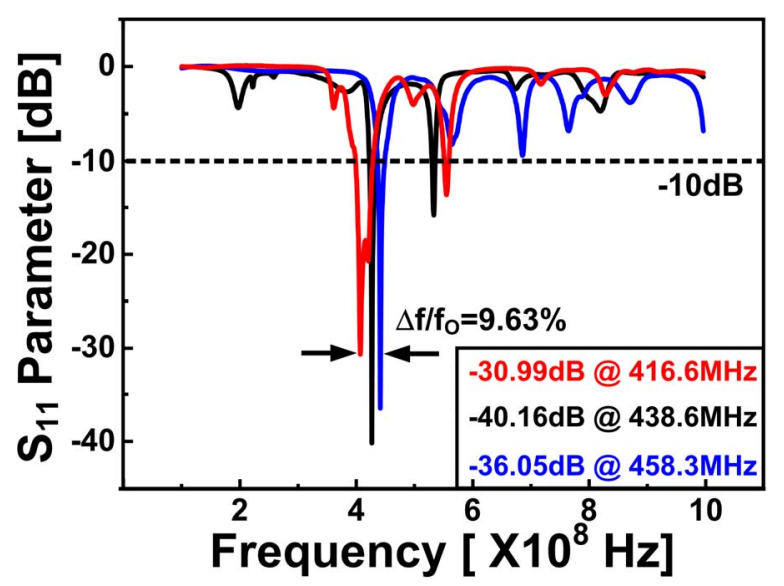

Figure 3. Measured $\mathrm{S}_{11}$-parameter of the helical antenna

The stimulator chip consists of: 1) a zero-threshold NMOS rectifier [9] for high-sensitivity voltage rectification, 2) a reference and bias module for generation of regulated voltage, 3) a $5 \mathrm{~Hz}$ ring oscillator for determination of stimulation frequency, 4) and an APW stimulator to inject charge. Fig. 4 (a) shows the loss from the antenna made by conductive yarn. As the base station is away from EAs by $1 \mathrm{~m}$ and wirelessly provides power of $10 \mathrm{dBm}$, the required sensitivity for each EA is as low as $-16 \mathrm{dBm}$ as shown in Fig. 4 (c). A normal- $\mathrm{V}_{\text {th }}$

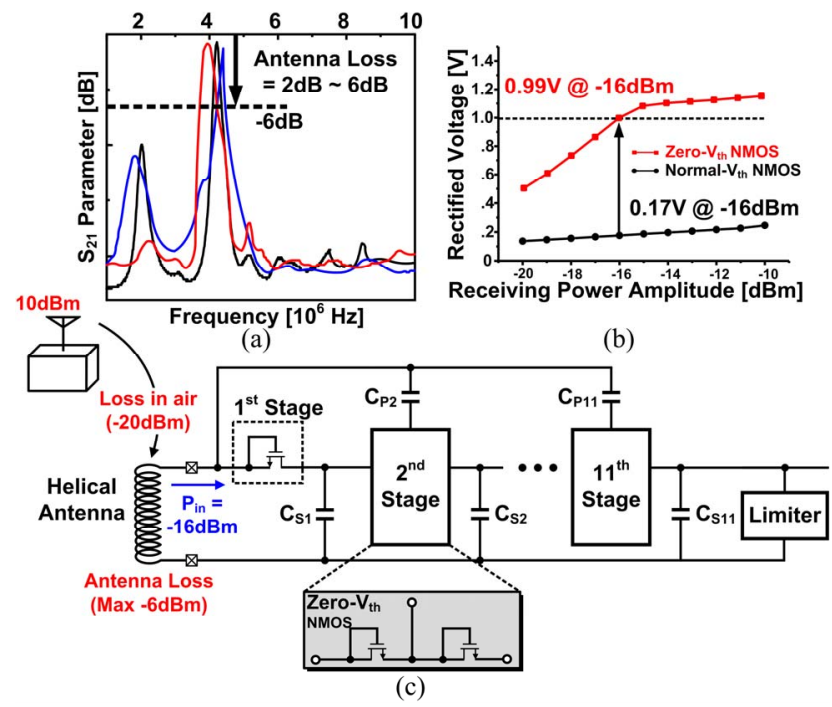

Figure 4. (a) Measured $\mathrm{S}_{21}$-parameter of the helical antenna,

(b) boosted voltage, and (c) the structure of zero- $\mathrm{V}_{\text {th }}$ NMOS rectifier

NMOS rectifier cannot satisfy the sensitivity of $-16 \mathrm{dBm}$, but a zero- $\mathrm{V}_{\text {th }}$ NMOS rectifier as shown in Fig. 4 (b). An 11-stage zero- $V_{\text {th }}$ NMOS rectifier (Fig. 4 (c)) is cascaded to generate required supply voltage of $1 \mathrm{~V}$ while withstanding the output current of $18 \mu \mathrm{A}$. The stable reference and bias are generated from the rectifier, and both operate a $5 \mathrm{~Hz}$ ring oscillator and reference the APW stimulator. The $5 \mathrm{~Hz}$ clock signal is shared with the external EA pair for Alternate Mono-Phase (AMP) stimulation.

\section{AdAPtive Pulse Width AND Alternate MonO- Phase Stimulation}

A. Adaptive Pulse Width (APW) Stimulator

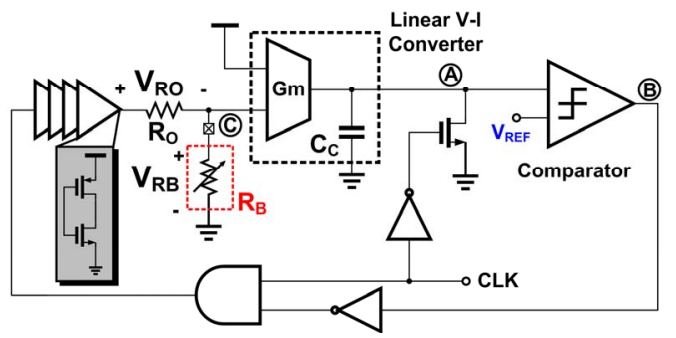

(a)

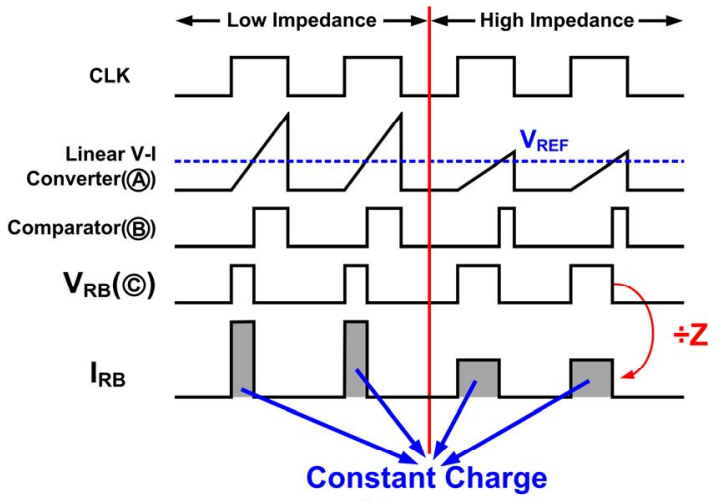

(b)

Figure 5. (a) The structure and (b) the time diagram of APW stimulator 
To achieve high immunity to BIV, the APW stimulator automatically generates a pulse-width-modulation (PWM) signal which is directly proportional to the BIV. Fig. 5 (a) shows the structure of APW stimulator. The operation of the proposed APW stimulator is divided into 4 steps. First of all, the BIV is detected by the on-chip sensing resistor of $R_{O}$ $(10 \mathrm{k} \Omega)$ to generate proportional voltage of $V_{R O}$. Secondly, transconductance amplifier $\left(G_{m}\right)$ with charging capacitor $C_{C}$ converts $V_{R O}$ into time domain slope signal. After that, the voltage stored at $C_{C}$ is compared with a reference voltage $\left(V_{R E F}\right)$ to generate PWM signal as shown in Fig. 5 (b). Meantime, clock signal is used to discharge $C_{C}$ for reset and synchronize mono-phase PWM signal of $V_{R B}$ which denotes the voltage across the $R_{B}$. As a result, a stimulating current pulse of $I_{R B}$, of which pulse width $\left(t_{P W}\right)$ in (1), is generated for constant charge $\left(Q_{I n j}\right)$ injection as shown in (2).

$$
\begin{gathered}
\frac{\left(G_{m} \cdot V_{R O}\right) \cdot t_{P W}}{C_{C}}=V_{R E F}, \text { Where, } t_{P W}=\frac{C_{C} \cdot V_{R E F}}{G_{m}} \cdot \frac{R_{B}}{R_{O} \cdot V_{D D}} \\
Q_{\text {Inj }}=I \cdot t_{P W}=\frac{C_{C} \cdot V_{R E F}}{G_{m} \cdot R_{O}}
\end{gathered}
$$

\section{B. Alternate Mono-Phase (AMP) Stimulation for Charge Balancing}

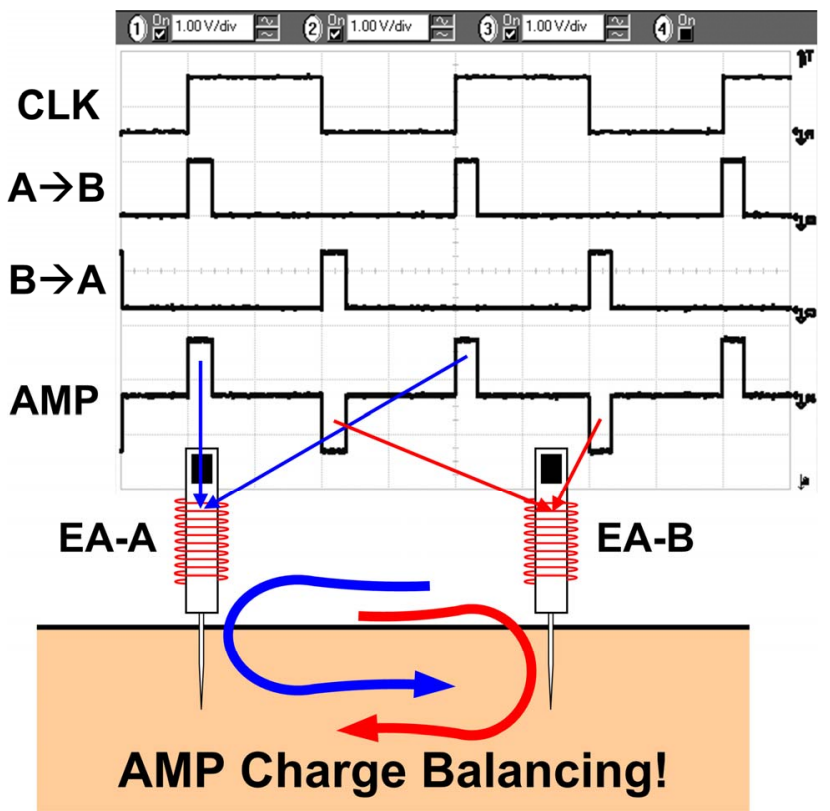

Figure 6. AMP stimulation scheme for charge balancing

Considering the safety of EA treatments, a bi-phase stimulation scheme is commonly used to neutralize the amount of injected charge into the body. However, it is impractical to be adopted in the wirelessly-powered EA environment; the generated supply voltage is as low as $1 \mathrm{~V}$, and the body-impedance of $200 \mathrm{k} \Omega$ limits the injected current level. To overcome this drawback, two EAs (EA-A, EA-B) are operated in alternate mono-phase fashion to achieve injected charge balancing as shown in Fig. 6. The EA-A operates as a master, which provides on-chip clock signal to EA-B (slave) for synchronization. At each clock rising edge, the injected current flows from EA-A to EA-B. On the contrary, at each clock falling edge, the current direction is swapped from EA$\mathrm{B}$ to EA-A. The differential current between EA-A and EA-B balances the amounts of injected charge so that AMP is accomplished by the repeated operation of every clock cycle.

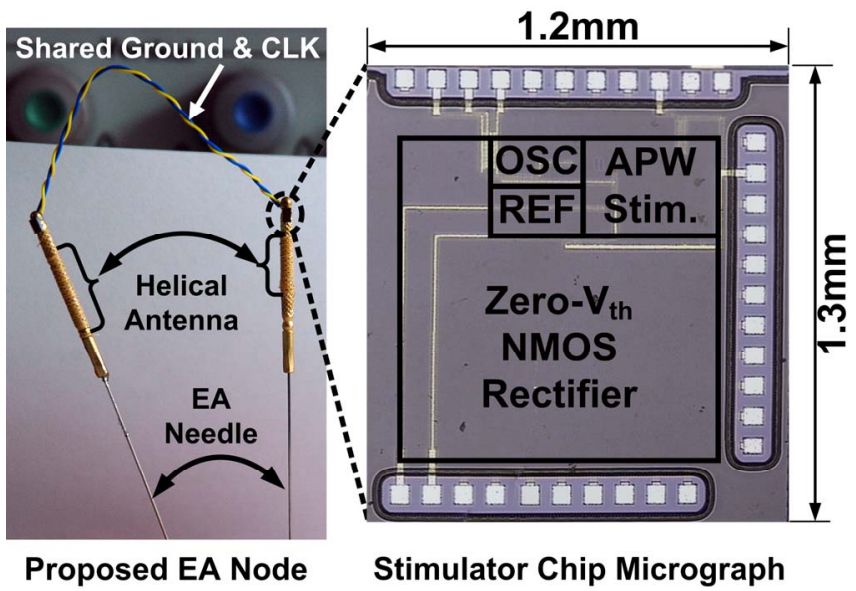

Figure 7. The proposed EA node with stimulator chip micrograph

\section{IMPLEMENTATION RESULTS}

Fig. 7 shows the implemented wirelessly-powered EA node with a $1.2 \mathrm{~mm} \times 1.3 \mathrm{~mm}$ stimulated chip which is fabricated in $0.18 \mu \mathrm{m} 1 \mathrm{P} 6 \mathrm{M}$ CMOS process. The stimulator chip is attached on the head of each needle, and the helical RF antenna is coiled around the needle just below the stimulator chip.

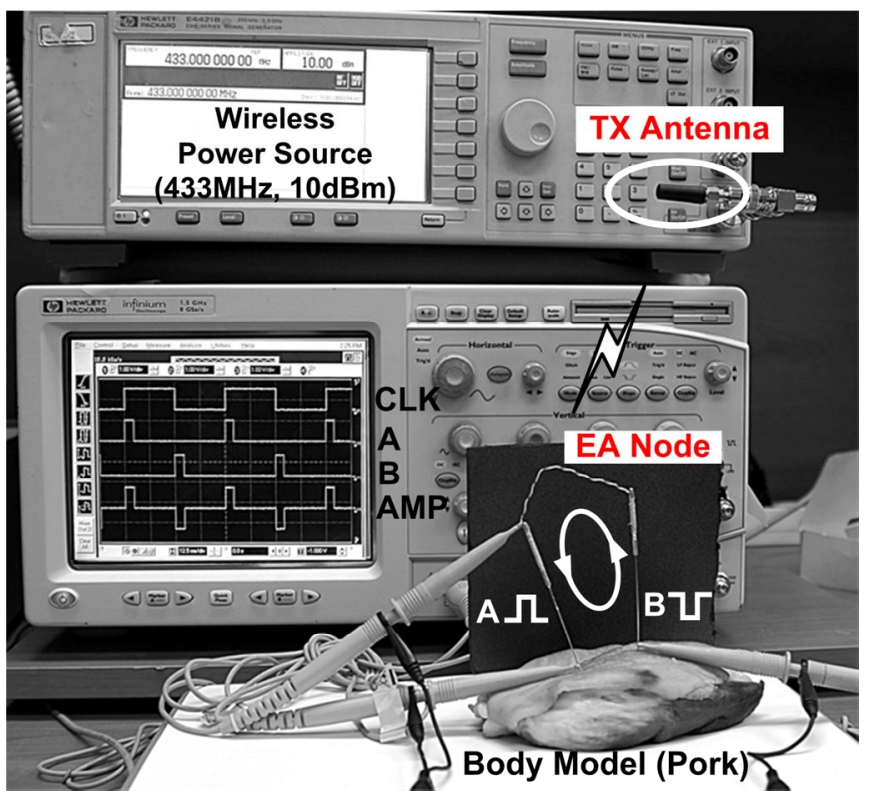

Figure 8 . The verification of proposed wirelessly-powered EA 
Fig. 8 shows the measurement setup of the wirelesslypowered EA system. The operation is verified as follows: 1) the RF signal generator transmits $433 \mathrm{MHz}-\mathrm{ISM}$ band $10 \mathrm{dBm}$ RF signal to the proposed EA node by a mono-pole antenna, 2) the proposed EA node generates supply voltage and injects charge in AMP fashion into a body model, and 3) the charge balanced pulses, independent of the BIV, are measured by an oscilloscope.

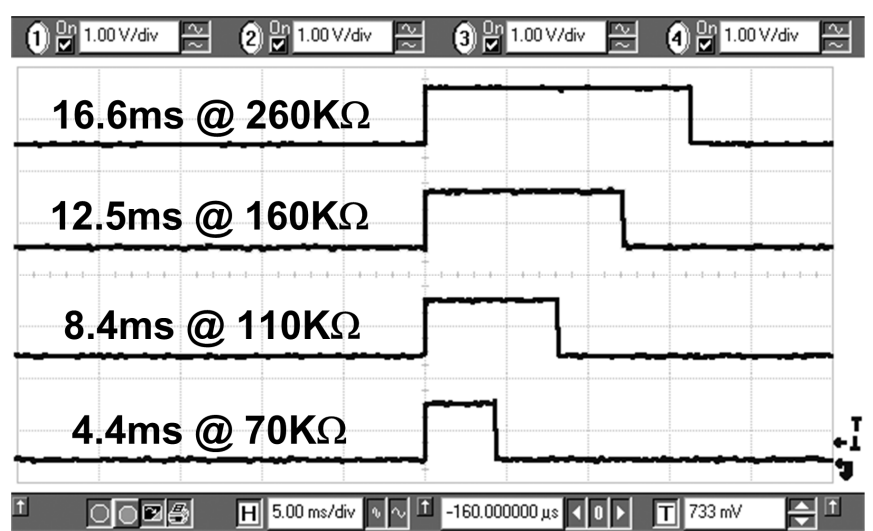

(a)

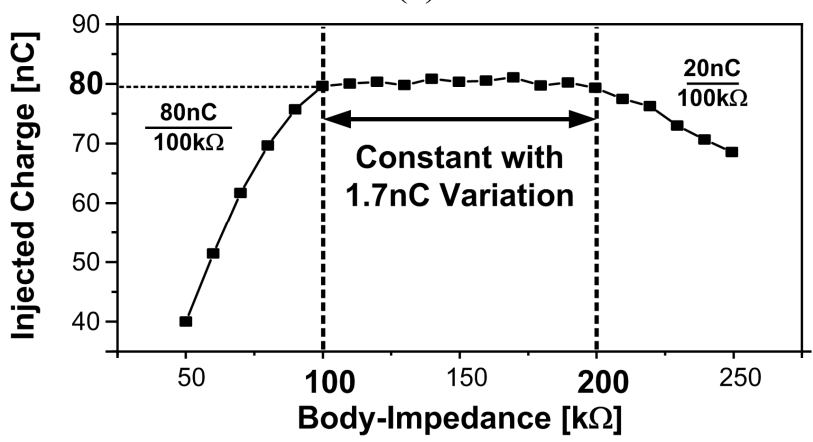

(b)

Figure. 9 (a) Measurement waveform of APW stimulator and (b) tolerable BIV range to maintain constant charge injection

Fig. 9 shows the measurement result of the APW stimulator and tolerable BIV range which can maintain constant charge injection. As shown in Fig. 9 (a), the pulse width is directly proportional to the body-impedance, and it equals to constant $80 \mathrm{nC}$ for stimulation. According to Fig. 9 (b), the proposed APW stimulator operates linearly with respect to BIV in the range $100 \mathrm{k} \Omega-200 \mathrm{k} \Omega$. In this range, the maximum deviation of injected charge level is within $1.7 \mathrm{nC}$. Table I summarizes the performance of the proposed wirelessly-powered EA.

\section{CONCLUSION}

In this paper, we propose a wirelessly-powered ElectroAcupuncture (EA). A tiny and light conductive yarn coiled around acupuncture eliminates cumbersome wire connection between EAs and EA base station. It also improves both patient's convenience and remedial value by the elimination of wire-connections and potential extension of EA channels. The APW stimulator is introduced to inject constant $80 \mathrm{nC}$ regardless of BIV in the range of $100 \mathrm{~K} \Omega-200 \mathrm{~K} \Omega$. The AMP guarantees the patient's safety by neutralization of the injected charge. The proposed wirelessly-powered EA node is verified by APW-AMP stimulation over body model against $-16 \mathrm{dBm}$ sensitivity at $433 \mathrm{MHz}-\mathrm{ISM}$ band.

TABLE I. PERFORMANCE SUMMARY

\begin{tabular}{|c|c|}
\hline Process & Samsung $0.18 \mu \mathrm{m}$ 1P6M CMOS \\
\hline Die Size & $1.2 \mathrm{~mm} \mathrm{X} 1.3 \mathrm{~mm}$ (Including Pads) \\
\hline Supply Voltage & $\begin{array}{c}1.0 \mathrm{~V} \\
1 \mathrm{~m} \text { away from EA Base Station } \\
\left(433 \mathrm{MHz}, \mathrm{P}_{\text {out }}=10 \mathrm{dBm}\right)\end{array}$ \\
\hline Injection Charge & $\begin{array}{c}\text { 80nC per stimulation } \\
\text { against } 100 \mathrm{k} \Omega-200 \mathrm{k} \Omega \text { BIV by APW }\end{array}$ \\
\hline Charge Balancing & Alternate Mono-Phase Stimulation \\
\hline $\begin{array}{l}\text { Stimulation } \\
\text { Frequency }\end{array}$ & $5 \mathrm{~Hz}$ \\
\hline Rectifier Type & $\begin{array}{l}\text { Zero-Threshold NMOS Rectifier } \\
\left(30.0 \% \mathrm{PCE} @ \mathrm{P}_{\mathrm{in}}=-12 \mathrm{dBm}\right)\end{array}$ \\
\hline Antenna & $\begin{array}{l}\text { Helical Antenna using Conductive Yarn } \\
\text { ( } 70 \text { turns, } 1 \mathrm{~mm} \text { diameter) }\end{array}$ \\
\hline
\end{tabular}

\section{REFERENCES}

[1] C. Deluze, L. Bosia, A. Zirbs, A. Chantraine, and T. L. Vischer, "Electroacupuncture in fibromyalgia: results of a controlled trial," www.bmj.com, accessed on : Oct. 2009.

[2] E. Stener-Victorin, U. Waldenstrom, S. A. Andersson and M. Wikland, "Reduction of blood flow impedance in the uterine arteries of infertile women with electro-acupuncture," European Society for Human Reproduction and Embryology Human Reproduction, vol.11, no.6, pp. 1314-1317, 1996.

[3] E. Stener-Victorin, U. Waldenstrom, L. Nilsson, M. Wikland and P. O. Janson, "A prospective randomized study of electro-acupuncture versus alfentanil as anaesthesia during oocyte aspiration in in-vitro fertilization," European Society of Human Reproduction and Embryology Human Reproduction, vol.14, no.10, pp. 2480-2484, 1999

[4] D. F Mayor, Electroacupuncture A practical manual and resource, $1^{\text {st }}$ ed., vol.1, Churchill Libingstone Elsevier, 2007.

[5] S. K. Kelly and J. Wyatt, "A power-efficient voltage-based neural tissue stimulator with energy recovery," in ISSCC Dig. Tech. Papers, pp. 228-524, Feb. 2004.

[6] W. Liu, "Retinal prothesis", in ISSCC Dig. Tech. Papers, pp. 218-219, Feb. 2004.

[7] M. Ortmanns, A. Rocke, M. Gehrke, H.-J. Tiedtke, "A neuro-stimulus chip with telemetry unit for retinal prosthesis device," IEEE Journal of Solid-State Circuits, vol. 35, no. 10, pp. 1487-1497, Oct. 2007.

[8] J. Yoo, S. Lee, and H.-J. Yoo, “A $1.12 \mathrm{pJ} / \mathrm{b}$ inductive transceiver with a fault-tolerant network switch for multi-layer wearable body area network applications," IEEE Journal of Solid-State Circuits, vol. 44, no. 11, Nov. 2009. (Accepted for publication)

[9] Y. Yao, J. Wu, Y. Shi, and F. F. Dai, "A fully integrated 900-MHz passive RFID transponder front end with novel zero-threshold RF-DC rectifier," IEEE Trans. on Ind. Electron., vol. 56, no. 7, pp. 2317-2325, Jul. 2009. 